\title{
COR TRIATRIATUM: CONGENITAL STENOSIS OF THE COMMON PULMONARY VEIN
}

\author{
BY \\ FRANK W. NASH and DONALD MACKINNON \\ From Aberdare General Hospital
}

(RECEIVED FOR PUblicatioN JANUARY 12, 1956)

Cor triatriatum is the term given to a rare congenital abnormality of the heart in which a septum separates what is apparently one of the atria into upper posterior and lower anterior chambers. With few exceptions, the septum has always been present on the left side of the heart. Other names given to the same anomaly naturally reflect the authors' views concerning the nature and origin of the extra chamber. Thus Loeffler (1949) reported his case as a 'heart with pulmonary sinus' and Edwards, Du Shane, Alcott and Burchell (1951) have referred to 'stenosis of the common pulmonary vein'.

From the functional aspect, three varieties of the condition have been described (Loeffler, 1949). In one, there is no communication between the accessory chamber and the left atrium. Death is inevitable immediately after delivery unless there is some other anomaly allowing blood to escape from the accessory chamber as in Case 3 of Edwards et al. (1951). At the other extreme there is an opening in the septum which is relatively large so that there is little or no obstruction to the flow of blood through the left side of the heart as in the patients of Loeffler (1949) and of Griffith (1903). In the intermediate variety the communication between the accessory chamber and the left atrium consists of one or more small openings which offer considerable obstruction to blood flow.

The following is the report of a patient who comes into this last category.

\section{Case Report}

J.B., a male infant, aged 7 weeks, was admitted to hospital on July 15,1954 . Pregnancy had been uneventful and the infant was delivered at full term after a normal labour. The birth weight was $7 \mathrm{lb} .4 \mathrm{oz}$. Both parents and an older child aged 3 years were in good health. The child appeared normal at birth and thrived satisfactorily until the onset of the present illness. Five days before admission to hospital he developed an irritant cough and began to vomit his feeds. These symptoms became progressively worse and he was therefore referred to hospital. On admission, he was slightly cyanosed and had signs of consolidation at the right lung base: temperature $97^{\circ}$ F.: pulse 150/minute, regular: respirations 60 /minute. The heart was not clinically enlarged and no murmur could be heard. The other systems were normal. The infant was nursed in oxygen and given penicillin parenterally and sulphadimidine by mouth. Initially there was some improvement but a few days after admission obvious signs of congestive heart failure developed; the liver enlarged rapidly and pitting oedema of the sacrum, buttocks and legs appeared. Treatment with 'digoxin' was begun but the congestive heart failure responded only temporarily and the patient died 13 days after admission.

Investigations during life showed that the urine contained a trace of albumin, but on microscopy was normal.

A radiograph of the chest (portable film) showed apparent enlargement of the heart. There was bronchopneumonic shadowing at the right lung base. The left lung was largely obscured by the heart shadow. Unfortunately, an electrocardiogram was not taken, and the child was considered too ill for heart screening.

\section{Necropsy}

The pathologist's report is as follows:

'The body shows no external abnormalities and is well nourished. The heart is enlarged and weighs $43 \mathrm{~g}$. The enlargement is due to right ventricular hypertrophy. The right atrium is dilated and communicates normally with the superior vena cava and inferior vena cava. The foramen ovale is patent and the opening measures $5 \mathrm{~mm}$. The left ventricle is small and there is no interventricular septal defect. The left atrium is divided by a septum into two chambers, postero-superior and antero-inferior. These communicate by a small opening in the septum, approximately $1 \mathrm{~mm}$. in diameter, which is situated just above the posterior cusp of the mitral valve. The upper chamber or supernumerary atrium receives three pulmonary veins, two on the right side and one on the left. The lower chamber has the foramen ovale in its medial wall and communicates with the left ventricle through a normal mitral orifice. The left auricle forms part of the lower chamber. A drawing of the specimen is reproduced in Fig. 1. 


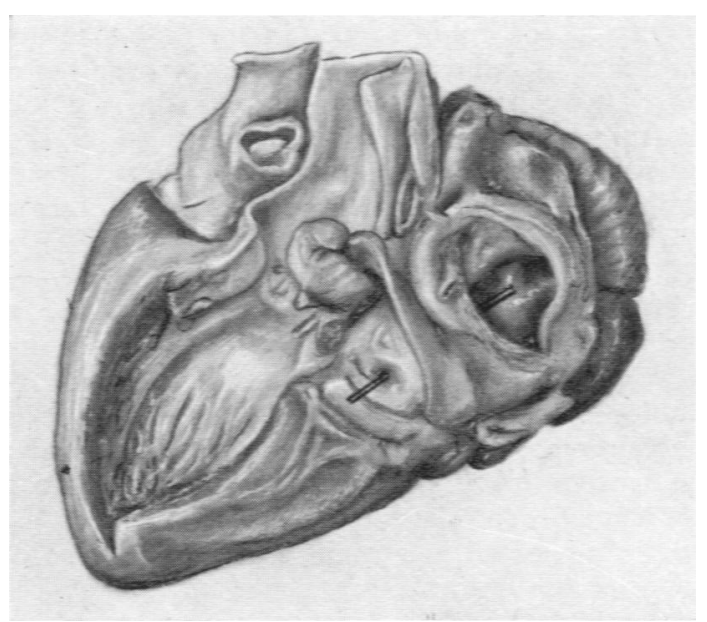

FIG. 1-A drawing of the left antero-lateral aspect of the heart showing a probe passing through a small opening in the septum which separates the upper and lower chambers. The latter communicates with a small left ventricle through a normal mitral orifice.

The other organs appear anatomically normal and show the changes of chronic venous congestion.

'Microscopic examination of the heart shows that the muscle is normal and there is no evidence of fibroelastosis. The septum dividing the left atrium into two chambers is composed of fibrous tissue and cardiac muscle, and the upper and lower surfaces are covered by endothelium. The lungs, liver and spleen show the features of chronic venous congestion.'

\section{Discussion}

The heart lesion discovered in our patient is undoubtedly very rare. Maud Abbott (1936), in an analysis of 1,000 necropsies of congenital heart disease, mentions 14 cases in which atrial septa were present and were considered to be the primary lesion responsible for death. In 10 , the septum was in the left atrium (age range, birth-48 years; mean, 20 years; four males and six females).

Pedersen and Therkelsen (1954) have reviewed the reports of 19 cases of left-sided cor triatriatum in the world literature and added one of their own. They also found references to three other cases in which clinical and pathological data were lacking. Subsequent to their publication, there have been reports of two other cases (Haarscher, Marcoux and Levy, 1954; Hartmann, 1955).

The following short summary includes the relevant points concerning our own patient, and therefore relates to 23 cases in all. The data contained in Abbott's (1936) publication have been excluded, since the sources of her cases are not given, and some of them are almost certainly included in Pedersen and Therkelsen's review.
Age. Most of the patients have died in infancy or in the first few years of life. Thus, of the 23 examples of the condition, 17 patients died before the age of 12 years, and of these nine died within the first six months of life. The age at death is not recorded in one patient. In all the childhood deaths, the opening in the septum was less than $6 \mathrm{~mm}$. in diameter. Those patients who reached adult life had relatively large septal openings (Church, 1868; Fowler, 1881-82; Preisz, 1889), with the exception of Pedersen and Therkelsen's patient (see below), a woman who lived until the age of 29 years and in whom the orifice in the septum was estimated at 5-7 $\mathrm{mm}$. in diameter.

Sex. The sex incidence is equal; of 21 cases in which the sex is recorded, 10 were males and 11 females.

Clinical Features. In some of the patients progress had been normal until shortly before death, as in the present child, and the terminal feature had been a rapidly developing congestive heart failure. In others, as in many examples of congenital heart disease, recurrent respiratory infections had been encountered. Often there is no definite cardiac enlargement on clinical examination and in several patients no heart murmurs have been heard. Tachycardia is a common feature and this may well obscure any murmur that might be present at slower rates. Other signs include those of bronchopneumonia and of right-sided heart failure. Electrocardiograms were recorded in five patients only, and in all right axis deviation was present together with signs of right ventricular hypertrophy. The patient described by Pedersen and Therkelsen (1954) seems to be the only one in whom detailed cardiovascular studies were made before death. These authors recorded the details of a 29-year-old woman suffering from increasing cardiac distress and in whom radiological, electrocardiographic and catheterization studies pointed to pulmonary hypertension due to obstruction in the venous side of the lesser circuit. Thoracotomy was undertaken in order to relieve what was presumed to be mitral stenosis, but a normal mitral valve was encountered. At necropsy the typical findings of cor triatriatum were discovered.

Angiocardiography has not yet been employed in any of these patients.

Morbid Anatomy. The usual picture is that of a diaphragm which separates the left atrium into an upper posterior chamber receiving the pulmonary veins, and a lower anterior one which contains the 
mitral orifice. The auricle always opens into the lower chamber. The septum is never more than a few millimetres in thickness and may be extremely thin. The perforation in the septum is usually single but there may be several. In two cases (Stöber, 1908; Tannenburg, 1930) the diaphragm was intact and the only exit of blood from the chamber was through an atrial septal defect in both, and also, in the first case, through anomalous pulmonary veins.

Other congenital defects are not frequent; they are usually in the nature of atrial septal defects which may communicate with either the upper or lower chambers of the left atrium.

Naked-eye and histological findings of endocardial fibro-elastosis have been noted in a few patients (Doxiadis and Emery, 1953).

The Nature of the Malformation. Several hypotheses have been put forward to explain the malformation in terms of embryology. These have been critically reviewed by Loeffler (1949) and Parsons (1950). The modern concept was anticipated many years ago by Griffith (1903) who suggested that there was 'a failure in the complete amalgamation of that part of the auricle which is said to be formed from the confluent portions of the pulmonary veins and that derived from the left hand division of the common auricle of the embryonic heart'. This has been put in a more concise way by Edwards et al. (1951), according to whom the malformation is a result of developmental arrest at the stage when the sino-atrial region of the heart develops the evagination which later becomes the common pulmonary vein, a process which has been demonstrated in the human embryo ( 2 to $7 \mathrm{~mm}$.) by Auër (1948). The accessory chamber, which receives the pulmonary veins, exists because of failure of the normal incorporation of the common pulmonary vein into the left atrium. It is thus a dilated common pulmonary vein, and the septal opening represents the junction of the sino-atrial portion of the heart with the embryonic common pulmonary vein. The septum is therefore formed by the posterior wall of the primitive left atrium and the inferior wall of the dilated common pulmonary vein.

Diagnosis. As yet no patient with cor triatriatum has been diagnosed in life; but it is not improbable that eventually this will be accomplished. The importance of doing so lies in the fact that this condition, if unaccompanied by other congenital heart anomalies, is, on theoretical grounds, suitable for surgical treatment. Without detailed investigation the diagnosis at best can be suspected in any patient with pulmonary hypertension of obscure origin and with electrocardiographic signs of right ventricular hypertrophy.

Probably the only certain way to establish the diagnosis in life would be to carry out an exploratory thoracotomy. Before cardiotomy, the demonstration of a high pressure in the pulmonary veins with a normal pressure in the left atrium would give the surgeon some indication of what to expect. As pointed out by Pedersen and Therkelsen, the surgical approach in opening the heart may be of importance. Entrance through the auricular appendage (auricle) would be distal to the membrane and, as in their patient, a normal mitral valve would be encountered. This may lead to a mistaken diagnosis. Where the diagnosis is suspected it is suggested that entrance through a pulmonary vein would be preferable.

\section{Summary}

The details are recorded of a male infant who died in congestive heart failure at the age of 9 weeks. Post-mortem examination showed that the heart was affected by an extremely rare anomaly, cor triatriatum, or congenital stenosis of the common pulmonary vein. Reference is made to previously reported examples of the condition and a short analysis of age and sex distribution, clinical features and morbid anatomy is based on a total of 23 cases in the literature. The nature of the developmental defect and the possibility of operative treatment are briefly discussed.

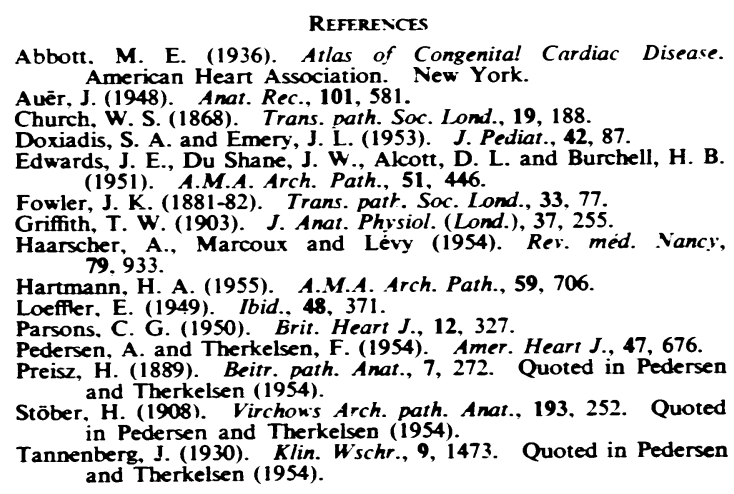
and Therkelsen (1954). 